\title{
Design of a Resistorless ASIC Preamplifier for HPGe Detectors with Non-Linear Pole/Zero Cancellation and Controlled Fast-Reset Feature
}

\author{
A. Pullia and S. Capra
}

\begin{abstract}
An original low-noise ASIC preamplifier is under development for high-resolution gamma-ray spectroscopy with germanium or other semiconductor detectors. The preamplifier is being designed in a $0.35 \mu \mathrm{m} 5 \mathrm{~V}$ CMOS technology, uses no feedback resistor and incorporates original techniques to optimize its performance. The first novelty consists of a nonstandard circuit structure for non-linear pole-zero compensation, aimed at removing the non-linear behaviour of the continuous non-resistive charge reset mechanism. The second consists of a controlled fast-reset feature aimed at greatly reducing the dead time caused by hits of high-energy charged particles. Such events are typical in nuclear physics experiments employing radioactive ion beams and in fusion-evaporation reactions which yield a prolonged saturation of the system that prevents detection of the subsequent decay events. The peculiar features of this preamplifier could be also exploited in other applications employing semiconductor detectors.
\end{abstract}

\section{INTRODUCTION}

$\mathrm{L}$ AST and next generation nuclear-physics experiments based ${ }_{\text {on }}$ radioactive ion beams and employing arrays of largevolume highly-segmented HPGe (High Purity Germanium) detectors [1]-[5] pose stringent requirements to the front-end electronics. One of the most critical issues is related to the foreseen background of charged particles, like pions or kaons of energies up to $100 \mathrm{MeV}$ or more. When these particles hit a detector crystal they are fully absorbed and generate huge charge signals that can easily put the front-end electronics in a prolonged saturation state, paralyzing the system and compromising its functionalities. So, it is of paramount importance that the front-end is capable to properly manage such large signals. Having said that, the front-end requirements include:

a) low noise

b) high linearity and no distortion of the exponentialdecay signal shape over the whole output voltage swing

c) reduced dead time in presence of large detector signals

d) low physical size and power consumption

This paper is focused on the design of an integrated Charge Sensitive Preamplifier (CSP) which addresses the shown issues utilizing original circuit solutions. The first general issue to be addressed is related to the unavailability of large-value

A. Pullia is with the Department of Physics, University of Milano, Italy, and INFN of Milano, Italy (email: alberto.pullia@mi.infn.it).

S. Capra is with the Department of Physics, University of Milano, Italy resistors in integrated circuit technologies. So the continuous reset of the charge signals cannot be made through the gigaohm feedback resistor typically employed in discretecomponent charge amplifiers and a resistorless solution is instead needed.

\section{PRIOR ART}

We took a known resistorless CSP [6] as a reference for this work. The original ASIC has been proposed and used for Xray fluorescence spectroscopy with silicon strip detectors, and proved to be extremely good in performance with a very low noise of 20 r.m.s. electrons. A simplified schematic diagram of such solution is shown in Fig. 1. The circuit uses no feedback resistor and auto sets its $\mathrm{DC}$ working point through a negative feedback path closed by a differential pair $\mathrm{T}_{1} / \mathrm{T}_{2}$. The two constant current generators "I" and "2I" provide the bias current to the differential pair. The shown operational amplifier was realized in CMOS technology and used a p-type MOSFET as input transistor. All technical details of the design can be found in [6]. Other useful examples can be found in [7][10]. If the detector current is much smaller than "I" then $T_{1}$ and $\mathrm{T}_{2}$ get biased at the same drain current and have identical transconductance $\mathrm{g}_{\mathrm{m}}$. It is easily found that in a first approximation the DC output voltage is

$v_{0_{-} D C}=V_{r e f}-i_{D_{-} D C} \frac{2}{g_{m}}$

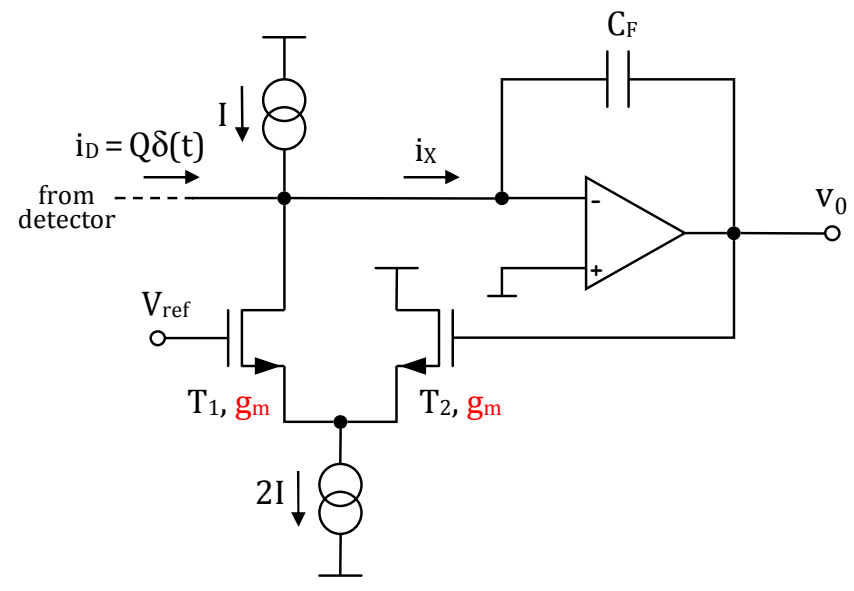

Fig.1. Prior art. 


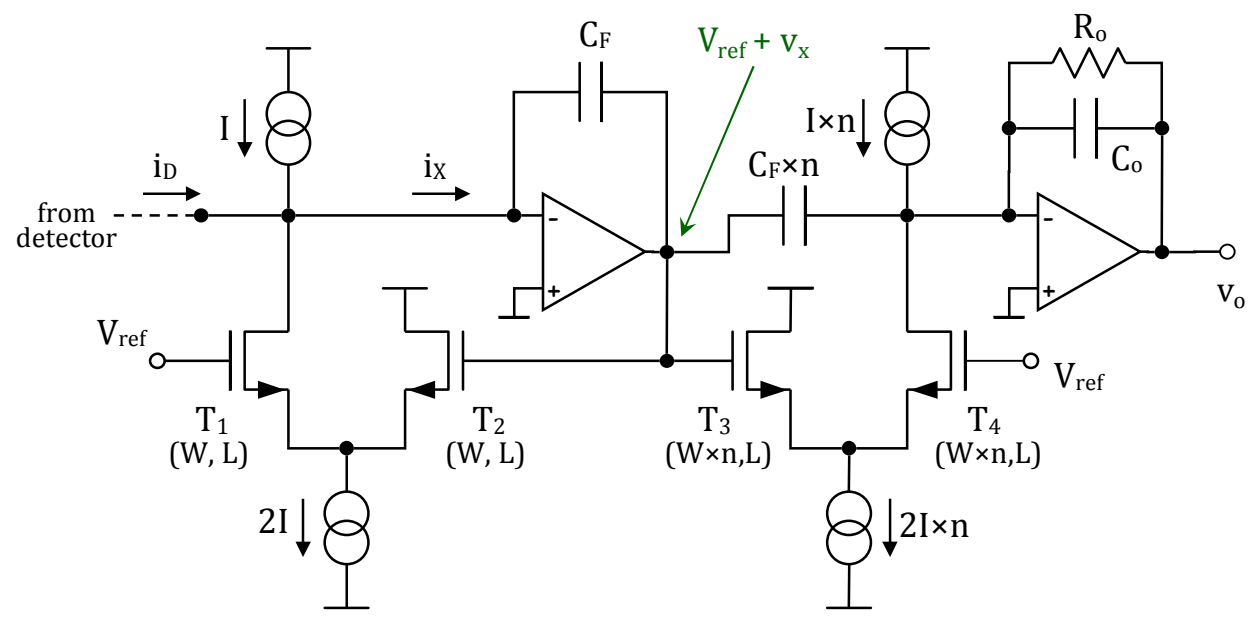

Fig.2. Circuit proposed in this paper for the cancellation of the non linearity in the response function for large signals. Non-linearity cancellation is obtained through a smart second stage employing a differential pair $T_{3} / T_{4}$ always working with the same gate, source and drain voltages as differential pair $T_{1} / T_{2}$. Notice that the transistors, the cross capacitance, and the constant current generators used in the second stage are scaled up in size of a factor $n$.

where $i_{D \_D C}$ is the detector leakage current and $g_{m}$ is the transconductance of $T_{1}$ and $T_{2}$. Notice that with no leakage current, or $\mathrm{i}_{\mathrm{DC}}=0$, the output voltage is equal to $\mathrm{V}_{\text {ref }}$. The range of detector leakage currents that this auto bias mechanism can work on is $\pm \mathrm{I}$. In this regard "I" should be chosen large. However current "I" should also be chosen as little as possible in order to minimize the input parallel noise. As a compromise "I" is typically set in the $100 \mathrm{pA}$ range. The transfer function of the circuit of Fig. 1 is easily calculated with a small-signal analysis in the frequency domain, which yields equations

$\left\{\begin{array}{l}I_{X}=I_{D}+V_{0} / \frac{2}{g_{m}} \\ V_{0}=-I_{X} \frac{1}{S C_{F}}\end{array}\right.$

where "s" is the independent variable in the Laplace domain and capitalized symbols are the Laplace counterparts of the non capitalized ones. Solving system (2) yields

$V_{0}=-\frac{I_{D}}{C_{F}} \frac{\frac{2}{g_{m}} C_{F}}{1+s C_{F} \frac{2}{g_{m}}}$

Notice that for $s \rightarrow 0$, i.e. at low frequency, (3) is in agreement with (1).

A major problem arises when the signals are not "small" and span over a large part of the output voltage swing, which is typical in gamma-ray spectroscopy. In fact the transfer function shown in (3) depends on $\mathrm{g}_{\mathrm{m}}$, which is a "small signal" parameter and can change quite a lot over the full voltage swing, which yields a non linear behavior and remarkable deviations from the expected signal shape. This cannot be tolerated in gamma spectroscopy and must be corrected.

\section{NON-LINEAR POLE-ZERO CANCELLATION}

In order to address this issue and so make the circuit of Fig. 1 adequate for gamma-ray spectroscopy we elaborated an original second-stage circuit structure able to remove the non linearity through a proper non-linear pole-zero cancellation. A similar technique has been first proposed by P. Rehak and coworkers [11] and then widely used worldwide. Our approach however is different and consists of the use of the second stage shown in Fig. 2. Differential pair $T_{3} / T_{4}$ is always working with the same gate, source and drain voltages as those of differential pair $T_{1} / T_{2}$, and is therefore affected by the same non linearity. Note also that the transistors, the cross capacitance, and the constant current generators used in the second stage are scaled up in size of a factor $n$. The configuration is such that all terms of (3) depending on parameter $g_{m}$ get cancelled off. Scaling up in size, moreover, yields a gain equal to $n$. With simple calculations one obtains

$I_{0}=V_{X} n \frac{g_{m}}{2}\left(1+s \frac{C_{F}}{\frac{g_{m}}{2}}\right)$

considering that $\mathrm{V}_{\mathrm{x}}$ in (4) corresponds to $\mathrm{V}_{0}$ in (3), and hence putting (3) in (4) one obtains

$I_{0}=-I_{D} n \frac{\frac{2}{g_{m}}}{1+s C_{F} \frac{2}{g_{m}}} \frac{g_{m}}{2}\left(1+s C_{F} \frac{2}{g_{m}}\right)=-I_{D} n$

where the pole and the zero as well as the individual $\mathrm{g}_{\mathrm{m}}$ terms cancel each other, which yields 


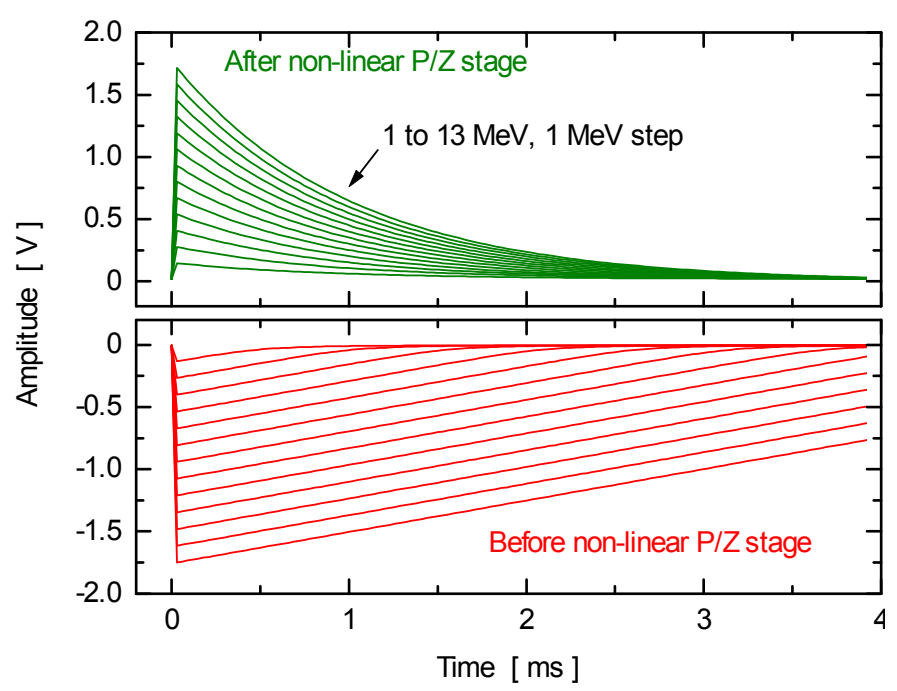

Fig.3. Computer simulation of the circuit response to a detector signal as seen at the first stage output (bottom) and at the second stage output (top). The "gain" term here is set to 1 . The effectiveness of the non-linearity cancellation is evident.

$V_{0}=I_{D} n \frac{R_{0}}{1+s C_{0} R_{0}}=\frac{I_{D}}{C_{F}}\left(n \frac{C_{F}}{C_{0}}\right) \frac{C_{0} R_{0}}{1+s C_{0} R_{0}}$

Now assuming that the signal delivered by the detector is delta like, i.e. $i_{D}=Q \delta(t)$ whose Laplace counterpart is $I_{D}=Q$, then (6) becomes

$V_{0}=\frac{Q}{C_{F}}\left(n \frac{C_{F}}{C_{0}}\right) \frac{C_{0} R_{0}}{1+s C_{0} R_{0}}$

Let us comment on (7):

- It does not depend on $\mathrm{g}_{\mathrm{m}}$, thanks to term-by-term cancellations shown in (5), which means that the non linearity brought about by $g_{m}$ is eliminated.

- Its time-domain counterpart is a clean exponential decay over the full available output voltage swing, as needed in gamma-ray spectroscopy

- It includes a "gain" term $n C_{F} / C_{0}$ that can further amplify the signal provided by the first stage, if needed

Fig. 3 shows a computer simulation of the circuit, where the "gain" factor has been set to one for simplicity. The signals seen at the first-stage output are shown on bottom. They have an irregular non-exponential shape caused by the circuit intrinsic non linearity which becomes evident when exceeding the limits of a small-signal operation. The very output signals are instead shown on top. In contrast they have a clean exponential-decay shape thanks to the non-linear pole-zero cancellation mechanism, as predicted by (7). The effectiveness of the non-linearity cancellation is thus very evident and very useful for the foreseen applications.

In Fig. 4 the plot is shown of the pulse heights of signals depicted in the upper part of Fig. 3 as a function of the event

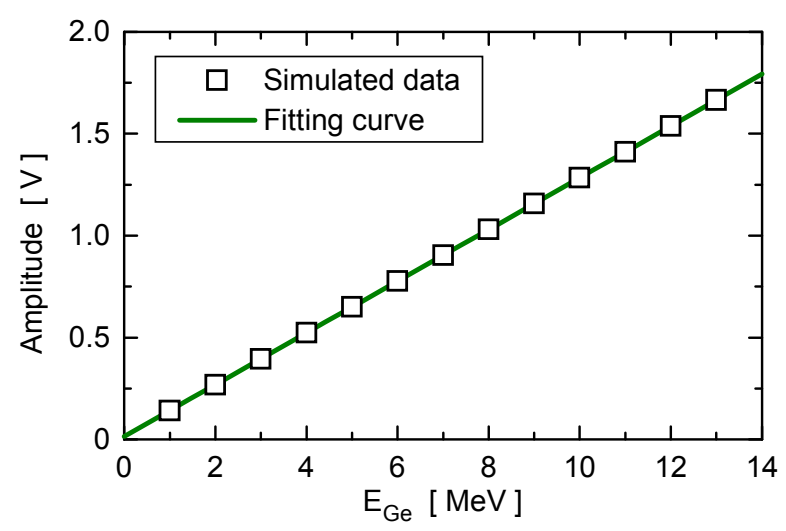

Fig. 4. Plot of the pulse heights of signals of upper Fig. 3 as a function of the event energy, assuming the energy-to-charge conversion of germanium. The relation is linear over the full output voltage swing.

energy, assuming the energy-to-charge conversion factor of germanium. As can be seen the relation is linear over the full voltage swing. So, system linearity is achieved both concerning the signal heights and concerning the signal shapes, which makes the circuit very adequate for gamma-ray spectroscopy.

It is worth mentioning that two or more such circuit structures could be cascaded for implementing a larger gain or/and for introducing more poles in the system transfer function and so obtain a signal shaping action with no use of large-value resistors. A Gaussian shaper with a shaping time in the $10 \mu$ s range, for example, could be easily implemented through a set of five or more properly-sized poles.

\section{CONTROLLED FAST-RESET CIRCUIT TECHNOLOGY (Patent Pending)}

The available circuit voltage swing as translated into energy for germanium detectors is $\sim 14 \mathrm{MeV}$, which is much less than the energy of the background charged particles mentioned earlier. Increasing the range to $100 \mathrm{MeV}$ or so with no impact on the signal-to-noise ratio would be possible with discrete component preamplifiers by using high power supplies of $\pm 12 \mathrm{~V}$ or $\pm 24 \mathrm{~V}$ [12]-[20], but is not with integrated solutions owing to the scaling down of all physical and electrical parameters. So the front end will necessarily enter a saturation state when such energetic particles hit the detector. To avoid that the inevitable saturation state paralyzes the system for a long time we propose the use of a "Controlled Fast Reset" circuit technology inspired to an old work of V. Radeka [21]. We discovered that such an approach also allows for precise measurement of the particle energy and we patented the related technique [22]. The circuit structure we used to implement the "Controlled Fast Reset" feature is shown in Fig. 5. As can be seen three devices are added to the CSP: a Schmitt-trigger comparator "A", a precise constant current sink " $\mathrm{I}_{R}$ ", and a double-throw electronic switch " $\mathrm{S}$ " driven by the comparator. When the amplitude of the signal is in the typical range of a few $\mathrm{MeV}$ the comparator is in its low logic state which sets the 


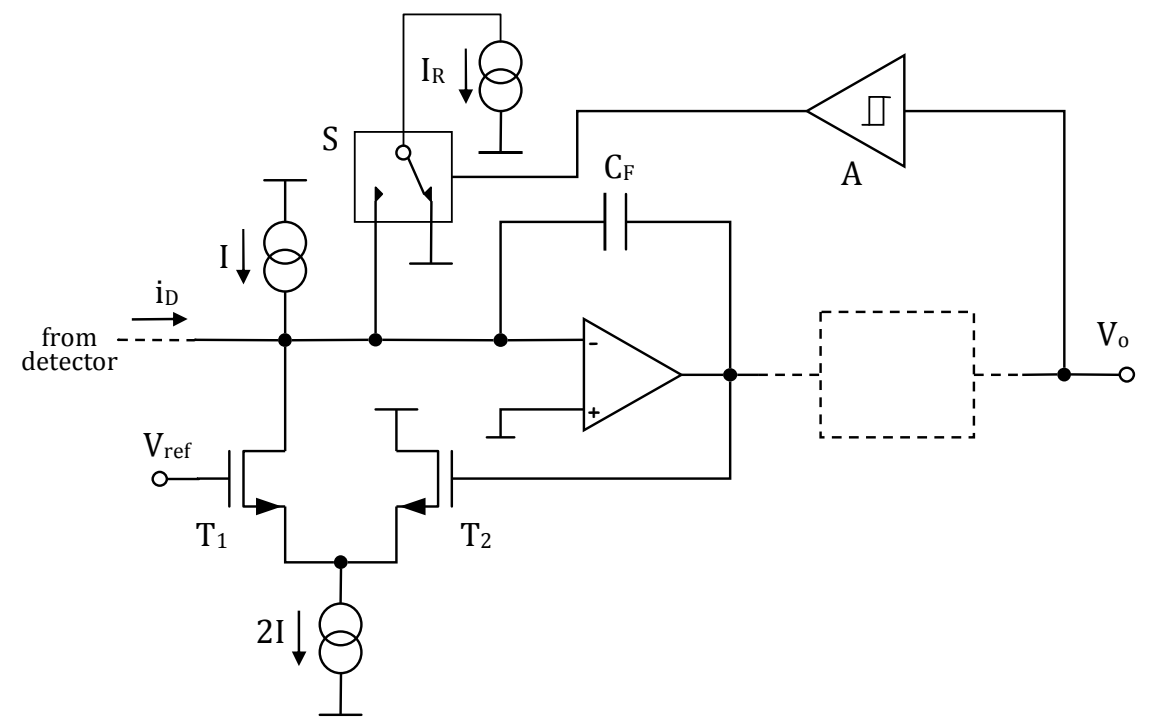

Fig.5. Simplified chematic diagram of the controlled reset circuit solution. The dashed block represents the circuitry used by the non-linear cancellation subsystem as given in detail in Fig. 2.

switch in the position shown in Fig. 5. In this phase the current sink does not interfere with the normal operation of the CSP. But when the signal overcomes the comparator threshold, the comparator goes to its high logic state, which drives a position change in the switch. The constant current generator $I_{R}$ now gets connected to the very input of the circuit and a controlled charge-removal process begins. Note that because " $I_{R}$ " is constant the charge removal rate is constant. We designate this as "controlled" reset process. The reset phase terminates automatically when the preamplifier output goes back to its baseline value of $0 \mathrm{~V}$. At this time the comparator commutates to its low logic state, which drives the switch back to its original position. The larger is " $I_{R}$ " the faster is the controlled reset. Overall this is an asynchronous pulsed reset. Typically

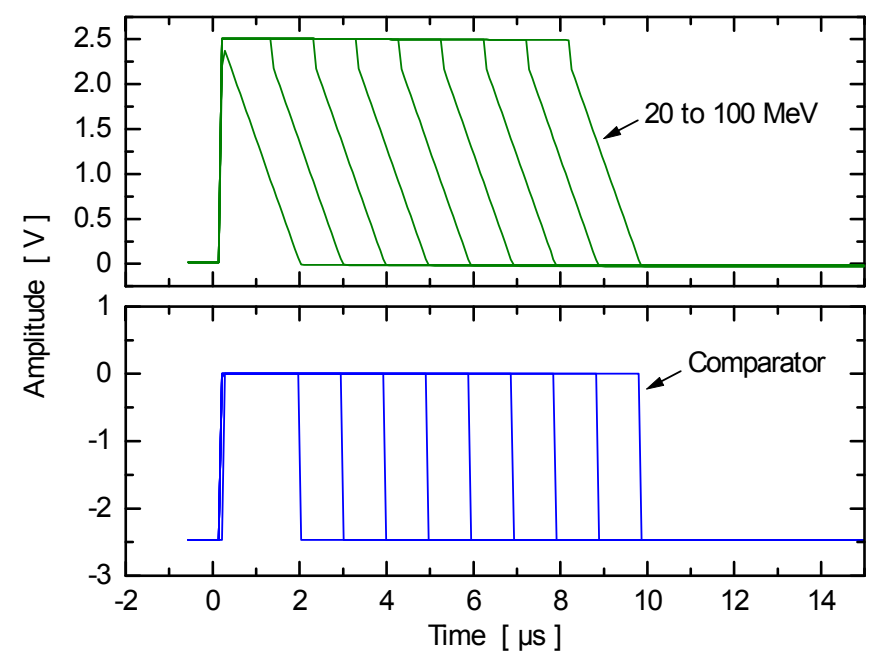

Fig.6. Computer simulation of the response of the controlled fast-reset preamplifier for input signals in the 20 to $100 \mathrm{MeV}$ energy range. On top the signals are shown as seen at the preamplifier output. On bottom the corresponding signals are shown as seen at the comparator output. we set " $I_{R}$ " in such a way that a signal of $100 \mathrm{MeV}$ gets fully reset in about $100 \mu \mathrm{s}$.

Fig. 6 shows on top the waveforms seen at the preamplifier output for input signals in the 20 to $100 \mathrm{MeV}$ energy range. The corresponding waveforms seen at the comparator output are shown on bottom. It can be seen that the Reset Time, as given by the width of the comparator signal grows regularly as the energy of the input signals is increased, which yields an indirect precise measurement of the signal energy. In Fig. 7 the plot is shown of the width of the comparator signal as a function of the event energy. It can be seen that the relation is highly linear irrespective of the fact that the preamplifier works in a highly non-linear saturated state. The shown plots are computer simulations but we have experimental data obtained with a simplified test chip which are in perfect agreement with the simulations and demonstrate the

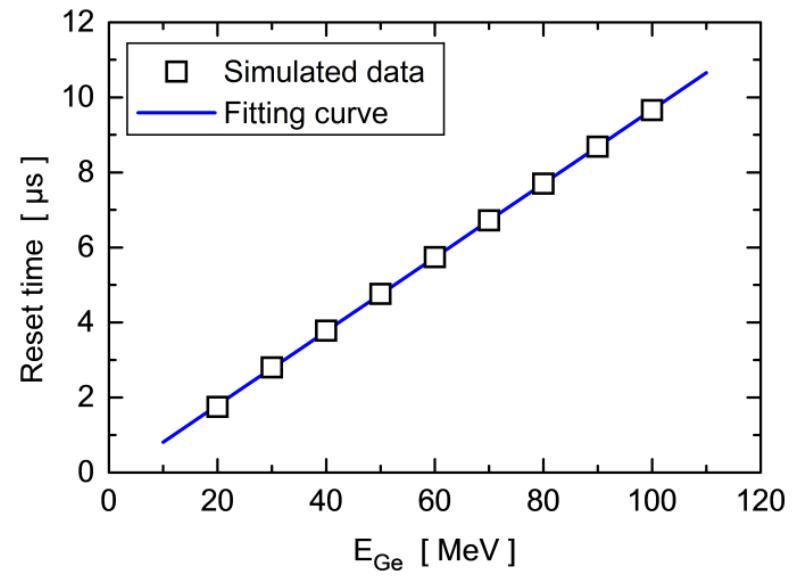

Fig.7. Plot of the widths of the comparator signals shown in lower Fig. 6 as a function of the event energy in the 20 to $100 \mathrm{MeV}$ range The deviation from linearity is below $\pm 0.1 \%$ over the whole range. 
effectiveness of this technology. Such data will be described in a dedicated paper. To the best of our knowledge this is the first time a linear measurement of the detector signal charge is performed with a front-end circuit working close to or in saturation, i.e. in a highly non linear state. This yields a huge extension of the linear measurement range. Combining the ranges of linear measurement of Figs. 4 and 7 an unprecedented dynamic range of $10^{5}$, i.e. from a few $\mathrm{keV}$ to more than $100 \mathrm{MeV}$, is achieved using a single front-end circuit. Moreover the controlled reset circuit is able to remove the large signal charge provided by the detector in a few $\mu \mathrm{s}$, which is a very short time as compared to the typical time constant of continuous reset preamplifiers in the ms range. So, whenever a large signal charge is generated by the detector the circuit removes it quickly and goes promptly back to its linear mode, where it is ready for further linear measurements.

\section{REFERENCES}

[1] AGATA homepage, URL: http://www-w2k.gsi.de/agata/

[2] S. Akkoyun et al., "AGATA-Advanced GAmmaTrackingArray", Nucl. Instrum. and Meth., vol. A 668, pp. 26-58, 2012

[3] GRETINA homepage, URL: http://grfs1.lbl.gov/

[4] A. Pullia et al., "Cross-Talk Limits of Highly Segmented Semiconductor Detectors", IEEE Trans. Nucl. Sci., vol. 58, no. 3, pp. 1201-1205, 2011

[5] A. Pullia et al., "Characterization of HPGe-Segmented Detectors from Noise Measurements", IEEE Trans. Nucl. Sci., vol. 51, no. 6, pp. 30863089, 2004

[6] B. Ludewigt et al, "A High Rate, Low Noise, X-Ray Silicon Strip Detector System", IEEE Trans. Nucl. Sci., vol. 41, no. 4, pp. 10371041, 1994

[7] A. Pullia et al, "Cryogenic Performance of a Low-Noise JFET-CMOS Preamplifier for HPGe Detectors", IEEE Trans. Nucl. Sci., vol. 57, no. 2, pp. 737-742, 2010

[8] A. Pullia et al., "A JFET-CMOS Fast Preamplifier for Segmented Germanium Detectors", IEEE Trans. Nucl. Sci., vol. 55, no. 1, pp. 591594, 2008
[9] A. Pullia et al., "A Compact VLSI dc Restorer for Multichannel X- $\gamma$ Ray Detectors", vol.. 52, no. 5, pp. 1643-1646, 2005

[10] C. Cattadori et al., "The GERmanium Detector Array read-out: Status and developments", Nucl. Instrum. and Meth., vol. A572, pp. 479-480, 2007

[11] P. Rehak et al., "Low-Noise CMOS Preamplifier-Shaper for Silicon Drift Detectors", IEEE Trans. Nucl. Sci., vol. 44, no. 3, pp. 385-388, 1997

[12] G. Pascovici et al., "Low noise, dual gain preamplifier with built in spectroscopic pulser for highly segmented high-purity germanium detectors", WSEAS Trans. on Circuits and Systems, vol. 7, no. 6, pp. 470-481, 2008

[13] A. Pullia and F. Zocca, "A low-noise preamplifier for $\gamma$-ray sensors with add-on device for large-signal management", Nucl. Instrum. and Meth., vol. A545, pp. 784-792, 2005

[14] A. Pullia et al., "An Advanced Preamplifier for Highly Segmented Germanium Detectors", IEEE Trans. Nucl. Sci., vol. 53, no. 5, pp. 2869-2875, 2006

[15] F. Zocca et al., "A Smart Reset Circuit for Low-Noise Preamplifiers of X- $\gamma$ Ray Sensor Signals", IEEE Trans. Nucl. Sci., vol. 54, no. 1, pp. 197-201, 2007

[16] F. Zocca et al., "A Time-Over-Threshold Technique for Wide Dynamic Range Gamma-Ray Spectroscopy With the AGATA Detector", IEEE Trans. Nucl. Sci., vol. 56, no. 4, pp. 2384-2391, 2009

[17] A. Pullia et al., "A 'cold' discharge mechanism for low-noise fast charge amplifiers", IEEE Trans. Nucl. Sci., vol.48, no.3, pp. 530-534, 2001

[18] A. Pullia et al., "Extending the dynamic range of nuclear pulse spectrometers", Rev. Sci. Instrum., vol. 79, p. 036105-01, 2008, doi: $10.1063 / 1.2894305$

[19] F. Zocca et al., "Design and Optimization of Low-Noise WideBandwidth Charge Preamplifiers for High Purity Germanium Detectors", IEEE Trans. Nucl. Sci., vol. 55, no. 2, pp. 695-702, 2008

[20] A. Pullia et al, "Low-Noise Amplification of $\gamma$-Ray Detector Signals in Hostile Environments", IEEE Trans. Nucl. Sci., vol. 53, no.3, pp. 17441748,2006

[21] V. Radeka, "Overload Recovery Circuit for Charge Amplifiers", IEEE Trans. Nucl. Sci., vol. 17, no. 1, p. 269, 1970

[22] A.Pullia and F. Zocca, "Technique for measuring detector signals", Italian Patent n. MI2012A001560, 19 Sept 2012 